\title{
Comparison of progression and non-progression in injecting drug users and homosexual men with documented dates of HIV-1 seroconversion
}

\section{Maria Prins and Paul J. Veugelers for the European Seroconverter Study and the Tricontinental Seroconverter Study}

Objective: To compare the progression and non-progression of HIV infection among 418 injecting drug users (IDU) and 422 homosexual men with documented dates of HIV seroconversion from 12 cohorts.

Methods: Seroconversion dates were calculated for each subject using a cohortspecific estimate of the cumulative HIV seroincidence over calendar time. In survival analysis, we studied the progression from seroconversion to AIDS and death by risk group. We compared non-progression between both risk groups by evaluating annual CD4 decline over the 7 years following seroconversion among AIDS-free subjects.

Results: The relative hazard ( $\mathrm{RH})$ of AIDS for homosexual men compared with IDU was 1.54 before, and 1.21 after, adjusting for age at seroconversion and year of seroconversion. The risk of death from any cause for homosexual men compared with IDU increased over time since seroconversion. Fifty IDU died prior to AIDS, compared with seven homosexual men (unadjusted $\mathrm{RH}$ for homosexual men 0.10). Ignoring this pre-AIDS mortality, the crude $\mathrm{RH}$ of death for homosexual men compared with IDU was 2.05. After adjusting for age at seroconversion and year of seroconversion in multivariate analysis, the $\mathrm{RH}$ became 1.42. No differences in progression between subgroups aged 24 years or older could be demonstrated, but subjects $<24$ years were found to be at a decreased risk. Proportions of nonprogressors based on CD4 slope $\geq 0$ at 7 years following seroconversion were higher for IDU than for homosexual men. No differences were found in the proportion $(\approx$ $5 \%$ ) classified as non-progressors by criteria of both slope $\geq 0$ and absolute CD4 counts $>500$ cells $\times 10^{6} / \mathrm{l}$, even if pre-AIDS deaths and losses to follow-up were included.

Conclusions: We found little evidence for an effect of risk group on progression and non-progression. Pre-AIDS mortality was much higher among IDU than homosexual men. Pre-AIDS mortality and a nonlinear age effect should be considered in planning interventions as well as studies comparing risk groups and modelling the epidemic.

AIDS 1997, 11:621-631

Keywords: Injecting drug users, homosexual men, HIV-seroconversion, progression, non-progression

From the Municipal Health Service, Division of Public Health and Environment, Amsterdam, The Netherlands.

Sponsorship: This research was supported by the Dutch AIDS Foundation (Stichting AIDS Fonds), as part of the Stimulation Program on AIDS Research of the Dutch Programme Committee for AIDS Research (94.008 and 1031) and by the grants of the original studies.

Requests for reprints to: Maria Prins, Municipal Health Service, Division of Public Health and Environment, Nieuwe Achtergracht 100, 1018 WT Amsterdam, The Netherlands.

This material was presented in part at the XI International Conference on AIDS, Vancouver, July 1996 (abstract number Tu.C.2520).

Date of receipt: 23 September 1996; revised: 6 January 1997; accepted: 14 January 1997. 


\section{Introduction}

Knowledge of risk group variation in the progression of HIV disease contributes to the understanding of the pathogenesis and may improve interventions. To date, most studies have evaluated risk group variation in seroprevalent cohorts. However, risk estimates obtained in these studies may be biased by the unknown duration of infection [1]. Hence, it is preferable to study disease progression in subjects for whom HIV seroconversion is documented. Studies that have been able to evaluate progression from HIV seroconversion to AIDS, immunological endpoints or death in both injecting drug users (IDU) and homosexual men [2-8] are limited. The drawbacks of some of these studies are the small numbers of subjects and limited durations of follow-up. Compared with cohort studies in homosexual men, most studies in IDU have started more recently and attrition is higher because of the lifestyle of this study population.

Results obtained from studies comparing IDU with homosexual men are not consistent. One study showed that progression rates in cohorts of IDU are slower than those in cohorts of homosexual men after adjusting for potential confounders such as age at seroconversion [9], while other studies found no differences [2-6,8].

Recently, various studies have attempted to characterize HIV-infected individuals who have not experienced clinical or immunological progression over a prolonged period of HIV infection in order to better understand the pathogenesis of the virus. The proportions of these non-progressors, based on data from established cohorts, range from $<1-25 \%$ [10-17] due to differences in study design, length of follow-up and inconsistency of the criteria used to define non-progression [16]. Nonetheless, since a long duration of follow-up is required, the most commonly studied risk groups are those that became infected first (i.e. homosexual men). To date, the potential differences in estimates of nonprogression between IDU and homosexual men have not been studied.

In this study, we compared IDU and homosexual men with respect to progression from HIV seroconversion to AIDS and death, and non-progression. We merged data from HIV-infected subjects with documented dates of seroconversion obtained from 12 cohorts of IDU or homosexual men. This large study group with a relatively long duration of follow-up has the potential to estimate risk group variation in the natural history of HIV infection more precisely and without the bias due to the unknown duration of infection.

\section{Materials and methods}

\section{Study population}

The HIV-positive study population comprised 418 IDU and 422 homosexual men in whom the dates of the last negative and first positive HIV-1 test were known.

An IDU is defined as a person who, since 1979, had injected drugs before HIV-seroconversion. The IDU originate from seven ongoing prospective studies registered in the European Seroconverter Study: the Edinburgh Drug Addiction Study and Edinburgh City Hospital Cohort Study (total number of seroconverters, 169); the Amsterdam Cohort Study among drug users ( $\mathrm{n}=99)$; the Geneva HIV cohort study $(\mathrm{n}=60)$; the French SEROCO (HIV-Seropositive Cohort Study) $(\mathrm{n}=38)$; the Scottish National Collaborative HIV testing Study from Glasgow $(\mathrm{n}=31)$, and the Innsbruck AIDS Study $(n=21)$. For IDU, the study population was composed first of IDU who entered the study HIV-negative and seroconverted during the follow-up (incident seroconverters, $\mathrm{n}=105$ ), and secondly IDU who were HIV-infected at entry to the study but who had earlier blood samples available to determine the interval of seroconversion (retrospectively identified seroconverters, $n=313$ ). For the latter, the blood samples were mostly obtained for hepatitis B serology in the early 1980s and for regular HIV-testing thereafter because of knowledge of risk behaviour.

Homosexual men originate from five cohorts registered in the Tricontinental Seroconverter Study: the Vancouver Lymphadenopathy-AIDS Study $(\mathrm{n}=132)$, the Sydney AIDS Prospective Study $(\mathrm{n}=79)$; the Amsterdam Cohort Study among homosexual men $(\mathrm{n}=146)$; the San Francisco General Hospital Study $(\mathrm{n}=19)$, and the San Francisco Men's Health Study $(n=46)$. For 397 of the 422 homosexual men, seroconversion was found prospectively. Twenty-five homosexual men of the Amsterdam cohort were seropositive at study entry, but had earlier seronegative blood samples as they had previously participated in a hepatitis $B$ vaccine trial.

In all cohorts, participants underwent standardized clinical examination, blood testing and completed questionnaires every 3-12 months. The design and methodology of each study have been described in detail elsewhere [18-25]. Information on AIDS diagnosis and the cause of death were obtained from review of medical records and/or through matching with local and national registers at each study location. In this analysis, AIDS was defined by the 1987 criteria of the Centres of Disease Control (CDC) [26].

Among the IDU, we combined the Glasgow, French and Innsbruck data because of the small number of sero- 
converters included in each cohort. Among the homosexual men, data for the two San Francisco cohorts were combined as in previous analyses $[16,25,27]$.

\section{Date of HIV seroconversion}

The estimated date of seroconversion is interval-censored by the last negative and the first positive HIV test. Since the risk of becoming HIV-infected may not be constant over this interval, basing analyses on the midpoint could result in substantially biased estimates of risk of progression, especially if seroconversion intervals are longer than 2 years [28]. In the present analysis, 127 IDU (30.4\%) and 45 homosexual men (10.7\%) had seroconversion intervals of more than 24 months. Therefore, the expected date of seroconversion was calculated for each of the 418 IDU and 422 homosexual men by the following procedure. First, we estimated the cumulative HIV seroincidence over calendar time for each cohort separately by applying the methods for interval-censored data $[29,30]$. Secondly, using the cohort-specific seroincidence distribution, the expected date of seroconversion was calculated for each subject conditional upon the date of each subject's last negative and first positive serological test.

On average, the estimated seroconversion dates were somewhat later than the midpoint between the last negative and the first positive test for IDU (i.e., increasing risk of becoming HIV-infected over the seroconversion interval) and somewhat earlier for homosexual men (i.e., decreasing risk), although there was some heterogeneity across the cohorts, as well as between the late and early seroconverters.

\section{Statistical analyses of progression}

We estimated the cumulative incidence of AIDS and death using Kaplan-Meier product-limit methods. The effect of risk group on progression was evaluated using Cox proportional hazards models. Entering risk group first, we evaluated various multivariate models to assess the effect of gender, age at seroconversion and date of seroconversion as covariates. Furthermore, we examined bias due to the length of the seroconversion interval and the interval between seroconversion and recruitment among retrospectively identified seroconverters. All these covariates had been evaluated within each risk group before pooled analyses of the data were performed.

To study the effect of risk group on progression stratification by site was only possible for the Amsterdam cohort since all seroconverters from the other sites belonged to one risk group. However, to evaluate whether the effect of risk group showed heterogeneity, we repeated the analysis replacing risk group with a dummy variable representing each cohort.

To evaluate the effect of gender, risk group was categorized as male IDU, female IDU and homosexual men.
Age at seroconversion and date of seroconversion were treated as continuous variables as well as categories. We tested for non-linear effects of the continuous variables by evaluating transformations. Categories of age at seroconversion were defined by cutoff points at the 25 th, 50th and 75th percentile of the total group (24, 29 and 35 years, respectively). Year of seroconversion was divided into the following five periods: before 1984, 1984-1985, 1986-1987, 1988-1989 and after 1989.

In Cox proportional hazards analysis, significance was determined by likelihood-ratio tests. We tested for interactions and verified the proportionality assumption for each covariate. The proportionality assumption was seriously violated for the effect of risk group on death from all causes. As shown by the survival curves in Fig. 1 , the risk of death from any cause for homosexual men compared with IDU increased over time since seroconversion. As this deviation is likely to be the result of differential pre-AIDS mortality [31], we studied progression from seroconversion to death separately for mortality prior to AIDS diagnosis (pre-AIDS death) and mortality with an AIDS diagnosis (death from AIDS). No other deviations from proportionality were found.

In survival analyses, the period of risk was defined as the interval between the estimated date of seroconversion and the date of the event (AIDS, pre-AIDS death, death from AIDS). Event-free subjects were censored at the last date with information on AIDS-free and vital status, respectively. Depending on the closing date of each data file merged and taking into account reporting delay of registries, the censoring date was ultimately 1 January 1995. For the analysis of progression to AIDS and death from AIDS, we censored all subjects who died without AIDS at the date of death. For the analysis of pre-AIDS mortality, follow-up was additionally censored at the date of diagnosis for subjects who developed AIDS.

Considering retrospectively-identified seroconverters at risk for AIDS and death at the date of seroconversion could underestimate progression rates if subjects who progressed or died rapidly after seroconversion were never recruited. Therefore, all retrospectively-identified seroconverters were left-truncated in survival analysis. For these seroconverters the date of enrolment was taken as the first date since seroconversion at which they were considered to be at risk for AIDS and death.

\section{Statistical analyses of non-progression}

One definition of non-progression, adapted from that of Muñoz et al. [12], was applied 7 years from seroconversion. In a previous evaluation of five nonprogression definitions within the Tricontinental Seroconverter Study [16], this definition was found to be consistent across cohorts and the most stable over 
time. The definition required subjects to be free of AIDS for 7 years and their annual CD4 decline based on a linear least-square regression line to be $>0$ (positive slope).

CD4 counts in the first year after seroconversion were excluded from the linear regression analysis, since CD4 counts are known to drop considerably immediately after seroconversion and more slowly thereafter $[32,33]$. AIDS-free subjects for whom no CD4 determinations were available in years $4-7$ and subjects with fewer than three CD4 determinations were considered lost to follow-up. Pre-AIDS deaths within 7 years from seroconversion were also considered lost to follow-up.

We calculated the proportion of subjects who were classified as non-progressors among all subjects who theoretically could have had at least 7 years of followup based on their estimated date of seroconversion, regardless of their clinical outcome. Seven years following seroconversion was chosen since this was close to the median follow-up time of both risk groups. Differences in proportions of non-progressors between risk groups were evaluated by Pearson's $\chi^{2}$ tests.

For all statistical tests, a $P$ value $<0.05$ was considered significant.

\section{Results}

The study group consisted of 418 IDU, including 156 (37.3\%) women, and 422 homosexual men. The median follow-up time since seroconversion was 6.3 years [interquartile range (IQR) 3.3-9.1 years] for IDU and 7.9 years (IQR 5.3-8.9) for homosexual men.

Characteristics of the study population stratified by cohort are displayed in Table 1 . The median age at seroconversion was 25.5 years among IDU, ranging from 23.1 years for the Edinburgh cohort to 29.7 years for the Amsterdam IDU cohort. For homosexual men, the median age at seroconversion was 33.8 years, ranging from 31.7 years for the Vancouver cohort to 35.6 years for the San Francisco cohort. The median interval between the last negative and the first positive HIV test was 1.14 and 0.54 years for IDU and homosexual men, respectively. For 313 IDU (74.9\%) and 25 homosexual men $(5.9 \%)$ seroconversion was determined retrospectively with a median lag time between seroconversion and enrolment of 1.6 (IQR 0.69-2.7) and 1.4 (IQR 0.97-2.8) years, respectively. On average, homosexual men were infected earlier than IDU. Seventy-six IDU and 169 homosexual men developed AIDS. Ninetyfive IDU and 137 homosexual men died, of whom 50 and seven died without AIDS in the respective groups.

\section{Progression to AIDS}

No statistically significant differences in progression from seroconversion to AIDS were found across IDU cohorts alone. The same was seen comparing the homosexual men cohorts (data not shown). The 7-year cumulative incidence of AIDS was 19.6\% [95\% confidence interval (CI) 15.0-25.3] for IDU and 33.8\% (95\% CI, 29.2-38.8) for homosexual men. For IDU, available follow-up was too short to estimate the median incubation period from seroconversion to AIDS using Kaplan-Meier methods. Using the current data and censoring strategy, the median incubation time for homosexual men by Kaplan-Meier estimates was 10.5

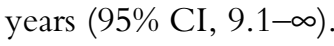

AIDS-defining conditions are presented in Table 2 . Most IDU (85.5\% of the AIDS cases) and homosexual men $(62.7 \%)$ had an AIDS-defining opportunistic infection. The homosexual men (25.4\%) were more likely to be diagnosed with Kaposi's sarcoma than IDU (1.3\%). Opportunistic infections and HIV encephalopathy were more frequent among IDU than among homosexual men.

Table 3 shows the results from Cox proportional hazards analysis. The risk of AIDS was 1.54-fold higher in homosexual men than IDU, but after adjusting for age at seroconversion and year of seroconversion the $\mathrm{RH}$

Table 1. Characteristics of 418 injecting drug users (IDU) and 422 homosexual men registered in the European Seroconverter Study and the Tricontinental Seroconverter Study, respectively, 1982-1995.

\begin{tabular}{|c|c|c|c|c|c|c|c|c|}
\hline Cohorts & $\mathrm{n}$ & $\begin{array}{c}\text { Age (years at } \\
\text { seroconversion) } \\
\text { median (IQR) }\end{array}$ & $\begin{array}{c}\text { Female } \\
\mathrm{n}(\%)\end{array}$ & $\begin{array}{c}\text { Seroconversion } \\
\text { interval (years)* } \\
\text { median (IQR) }\end{array}$ & $\begin{array}{l}\text { Calendar year } \\
\text { of seroconversion } \\
\text { median (IQR) }\end{array}$ & $\begin{array}{c}\text { AIDS } \\
\mathrm{n}\end{array}$ & $\begin{array}{c}\text { Deaths } \\
\mathrm{n}\end{array}$ & $\begin{array}{c}\text { Pre-AIDS } \\
\text { deaths } \\
n\end{array}$ \\
\hline IDU (total) & 418 & $25.5(22.2-29.8)$ & $156(37.3)$ & $1.14(0.39-2.4)$ & $87.2(84.2-90.0)$ & 76 & 95 & 50 \\
\hline Edinburgh & 169 & $23.1(20.4-27.9)$ & $57(33.7)$ & $1.33(0.52-2.4)$ & 84.1 (83.9-86.0) & 41 & 42 & 19 \\
\hline Amsterdam & 99 & $29.7(26.5-35.2)$ & $45(45.5)$ & $0.77(0.32-3.8)$ & $88.5(86.3-91.1)$ & 13 & 23 & 15 \\
\hline Geneva & 60 & $25.5(23.0-29.0)$ & $21(35.0)$ & $1.35(0.60-3.2)$ & $86.8(85.3-89.2)$ & 11 & 14 & 7 \\
\hline Other $^{+}$ & 90 & $24.7(22.3-28.0)$ & $33(36.7)$ & $0.91(0.35-1.6)$ & $89.8(88.8-92.0)$ & 11 & 16 & 9 \\
\hline Homosexual men (total) & 422 & $33.8(29.1-39.5)$ & & $0.54(0.27-.98)$ & 85.6 (84.9-87.3) & 169 & 137 & 7 \\
\hline Vancouver & 132 & $31.7(27.9-37.0)$ & & $0.84(0.58-1.17)$ & 85.1 (84.0-85.8) & 51 & 36 & 2 \\
\hline Sydney & 79 & $33.1(29.0-39.6)$ & & $0.53(0.25-0.94)$ & 85.6 (85.0-86.5) & 35 & 31 & 2 \\
\hline Amsterdam & 146 & $35.0(29.6-40.0)$ & & $0.27(0.25-0.50)$ & 86.3 (85.3-89.9) & 60 & 53 & 2 \\
\hline San Francisco & 65 & $35.6(32.0-41.7)$ & & $0.59(0.52-0.97)$ & $86.0(85.1-87.8)$ & 23 & 17 & 1 \\
\hline
\end{tabular}

*The interval between the date of the last negative and the first positive test. ${ }^{\dagger}$ The French SEROCO study $(n=38)$, the Scottish Collaborative HIV Testing Study $(n=31)$ and the Innsbruck AIDS Study $(n=21)$. IQR, Interquartile range. 
Table 2. AIDS-defining conditions among injecting drug users (IDU) and homosexual men registered in the European Seroconverter Study and the Tricontinental Seroconverter Study, respectively, 1982-1995

\begin{tabular}{|c|c|c|c|c|c|}
\hline & \multicolumn{2}{|c|}{$\begin{array}{l}\text { IDU diagnosed with AIDS } \\
\qquad(\mathrm{n}=76)\end{array}$} & \multicolumn{2}{|c|}{$\begin{array}{l}\text { Homosexual men diagnosed } \\
\text { with AIDS }(n=169)\end{array}$} & \multirow[b]{2}{*}{$P$ value ${ }^{\dagger}$} \\
\hline & $\mathrm{n}$ & $\% *$ & $\mathrm{n}$ & $\% *$ & \\
\hline $\begin{array}{l}>1 \text { AIDS-defining condition } \\
\text { Type of diagnosis }\end{array}$ & 5 & 6.6 & 15 & 8.9 & 0.72 \\
\hline Opportunistic infection & 65 & 85.5 & 106 & 62.7 & $<0.001$ \\
\hline Kaposi's sarcoma & 1 & 1.3 & 43 & 25.4 & $<0.001$ \\
\hline Other malignancy & 2 & 2.6 & 11 & 6.5 & 0.34 \\
\hline HIV encephalopathy & 9 & 11.8 & 4 & 2.4 & 0.006 \\
\hline Wasting syndrome & 1 & 1.3 & 10 & 5.9 & 0.20 \\
\hline
\end{tabular}

*Percentage among subjects diagnosed with AIDS. ${ }^{\dagger} P$ value by $\chi^{2}$ test comparing IDU with homosexual men. ${ }^{\ddagger}$ Two IDU and five homosexual men with $>1$ initial AIDS diagnosis of different categories are presented twice.

decreased to 1.21 and became non-significant $(95 \% \mathrm{CI}$, $0.87-1.67)$. Restricting the analysis to subjects with seroconversion intervals of less than 2 years did not change the RH for risk group. Since the risk of developing AIDS is known to be very small in the first year after seroconversion, we repeated the analysis for those who were recruited before or maximally one year after seroconversion. The adjusted RH for risk group hence became 1.63 .

The female IDU tended to progress slightly more slowly than male IDU (crude RH, 0.77), although the effect of gender was not significant. The risk of developing AIDS for homosexual men compared with male IDU was 1.41 (95\% CI, 1.03-1.92) before and 1.14 (95\% CI, 0.80-1.62) after adjusting for age at seroconversion and year of seroconversion.

As Kaposi's sarcoma is a very common and relatively early AIDS-diagnosis among homosexual men $[27,34,35]$, but is not common among IDU (Table 2), we re-analysed the data by considering subjects for whom only Kaposi's sarcoma defined AIDS lost to follow-up and AIDS-free at the date of Kaposi's sarcoma. When compared with the $\mathrm{RH}$ in Table 3, the crude $\mathrm{RH}$ for risk group decreased and did not reach statistical significance (RH, 1.20; 95\% CI, 0.90-1.59). When we adjusted for age at seroconversion and year of seroconversion, the progression rates became similar (RH, 0.94; 95\% CI, 0.67-1.32).

\section{Progression to death}

For IDU as well as homosexual men, the rates of progression to death did not differ significantly across cohorts (data not shown). The 7-year cumulative incidence of death from any cause was $23.6 \%(95 \% \mathrm{CI}$, 18.8-29.4) for IDU and $22.5 \%$ (95\% CI, 18.5-27.2) for homosexual men. For IDU, median time from seroconversion to death by Kaplan-Meier estimates was 10.7 years $(95 \% \mathrm{CI}, 10.0-\infty)$. For homosexual men the median time was 11.8 years (95\% CI, 10.6-12.0).

AIDS contributed to 45 deaths among IDU and 130 among homosexual men. Fifty IDU and seven homosexual men died without AIDS. Twenty-one of the 50 pre-AIDS deaths $(42.0 \%)$ among IDU and three of the

Table 3. Univariate and multivariate Cox proportional hazards analysis of progression from seroconversion to AIDS and death among injecting drug users (IDU) and homosexual men registered in the European Seroconverter Study and the Tricontinental Seroconverter Study, respectively.

\begin{tabular}{|c|c|c|c|}
\hline Cofactors & $\begin{array}{l}\text { AIDS } \\
\text { RH }(95 \% \mathrm{CI})\end{array}$ & $\begin{array}{l}\text { Pre-AIDS death } \\
\text { RH }(95 \% \mathrm{CI})\end{array}$ & $\begin{array}{l}\text { Death from AIDS } \\
\text { RH }(95 \% \mathrm{Cl})\end{array}$ \\
\hline \multicolumn{4}{|l|}{ Univariate analysis } \\
\hline \multicolumn{4}{|l|}{ Risk group } \\
\hline IDU & 1 & 1 & 1 \\
\hline homosexual men & $1.54(1.18-2.02)$ & $0.10(0.04-0.22)$ & $2.05(1.46-2.88)$ \\
\hline \multicolumn{4}{|l|}{ Multivariate analysis } \\
\hline \multicolumn{4}{|l|}{ Risk group } \\
\hline IDU & 1 & 1 & 1 \\
\hline homosexual men & $1.21(0.87-1.67)$ & $0.08(0.03-0.20)$ & $1.42(0.96-2.11)$ \\
\hline \multicolumn{4}{|c|}{ Age at seroconversion (years) } \\
\hline$<24$ & 1 & 1 & 1 \\
\hline $24-28$ & $2.34(1.51-3.63)$ & $1.25(0.64-2.44)$ & $3.23(1.80-5.79)$ \\
\hline $29-34$ & $1.78(1.12-2.81)$ & $0.64(0.25-1.63)$ & $2.45(1.34-4.49)$ \\
\hline$\geq 35$ years & $2.20(1.37-3.52)$ & $1.88(0.85-4.17)$ & $3.24(1.76-5.99)$ \\
\hline \multicolumn{4}{|l|}{ Year of seroconversion } \\
\hline before 1984 & 1 & 1 & 1 \\
\hline 1984-1985 & $1.00(0.70-1.42)$ & $1.81(0.83-3.97)$ & $1.20(0.78-1.86)$ \\
\hline 1986-1987 & $1.10(0.73-1.67)$ & $0.98(0.35-2.73)$ & $1.43(0.85-2.38)$ \\
\hline 1988-1989 & $0.85(0.47-1.55)$ & $1.11(0.37-3.32)$ & $1.53(0.71-3.31)$ \\
\hline After 1989 & $1.00(0.47-2.14)$ & $2.48(0.87-7.12)$ & $0.82(0.19-3.63)$ \\
\hline
\end{tabular}

$\mathrm{RH}$, Relative hazard; $\mathrm{Cl}$, confidence interval. 
seven pre-AIDS deaths (42.9\%) among homosexual men were attributed to an overdose or suicide. For three IDU (6.0\%) death was due to an accident or homicide. The remaining causes of death were nonAIDS (infectious) diseases $[\mathrm{n}=19(38.0 \%)$ among IDU; $\mathrm{n}=4(57.1 \%)$ among homosexual men] or unknown $[\mathrm{n}=7(14.0 \%)$ among IDU].

Since the hazard of risk group deviated from proportionality, we studied the progression to death by risk group separately for pre-AIDS death and death from AIDS. The crude and adjusted RH of pre-AIDS mortality (Table 3) showed that IDU were 10-fold more likely to experience pre-AIDS mortality than homosexual men. Ignoring this pre-AIDS mortality, the homosexual men were at greater risk for death than IDU. After adjusting for age at seroconversion and year of seroconversion in multivariate analysis, however, the $\mathrm{RH}$ of risk group for death from AIDS decreased and was of borderline significance. Restricting the analysis of time from seroconversion to death from AIDS to subjects with narrow seroconversion intervals, did not change RH substantially. Restricting the analysis to those who were recruited before and just after seroconversion resulted in a crude and adjusted $\mathrm{RH}$ for risk group of 2.40 and 1.74 , respectively.

Pre-AIDS mortality rates did not differ between male and female IDU. As observed for progression to AIDS, female IDU tended to progress more slowly to death from AIDS than male IDU (unadjusted RH, 0.59), although the effect was insignificant $(P=0.14)$. The RH for homosexual men compared with male IDU was $1.75(95 \%$ CI, 1.20-2.56) before and 1.26 (95\% $\mathrm{CI}, 0.82-1.92)$ after adjusting for age at seroconversion and year of seroconversion.

An age of less than 24 years was independently associated with slower progression to AIDS and death from AIDS (Table 3). The varying RH by increasing age categories (Table 3) indicates that the effect of age on progression was non-linear. This was confirmed by the fact that in the models that included age as a continuous variable and its quadratic term of age, the latter was significant. Since various transformations of age did not conform to the assumption of an exponential increase in relative hazard with concurrent increase in the continuous variable, we treated age as a categorical variable only.

We did not observe an effect of year of seroconversion on progression to AIDS and death from AIDS. Treating year of seroconversion as a continuous variable had a very minor influence on the presented $\mathrm{RH}$ of risk group. In an additional analysis, we evaluated the effect of calendar time (categorized as before 1991, 1992-1993 and > 1993) because certain events which occurred at different calendar times, such as the introduction of HIV treatment, might have influenced pro- gression rates. Including calendar period instead of year of seroconversion as a time-dependent covariate in the multivariate model displayed in Table 3, we did not find statistically significant trends over time and the RH for risk group did not change.

Models that included cohort as a covariate instead of risk group showed similar trends, with the IDU cohorts progressing slightly more slowly to AIDS and death from AIDS, but faster to pre-AIDS death, relative to cohorts of homosexual men. There was some heterogeneity for progression to AIDS and to death from AIDS, with the Geneva IDU cohort progressing slightly faster than the Vancouver homosexual men cohort, although differences were not statistically significant (data not shown).

No significant interactions among the variables in the multivariate models presented in Table 3 were found.

Because not all IDU cohorts registered in the European Seroconverter Study could match with AIDS or death registries, and to evaluate whether AIDS cases and deaths among cohorts who did match could have been missed, we reanalysed the data applying another censoring strategy. Subjects seen within 1 year before the ultimate date of censoring and not known to have progressed were considered event-free at the ultimate censoring date; subjects not seen within 1 year before the ultimate censoring date and not known to have progressed were censored at the date of their last visit; and finally, subjects known to have progressed were only regarded as such if the period between the last visit and the event did not exceed 2 years.

Figures 1 and 2 show the Kaplan-Meier estimates when this censoring strategy was applied. Compared with the results of the censoring strategy mentioned in Materials and methods, progression rates were only slightly slower for IDU. For homosexual men, progression was faster and the median incubation time from seroconversion to AIDS and death from AIDS became 9.0 years $(95 \% \mathrm{CI}, 8.0-9.9)$ and 10.6 years $(95 \% \mathrm{CI}$, 8.8-11.9), respectively. In comparison with the original censoring strategy, this strategy resulted in somewhat increased $\mathrm{RH}$ of AIDS and death from AIDS for homosexual men, relative to IDU (data not shown).

\section{Non-progression}

Two hundred and twenty-four out of 418 IDU and 339 out of 422 homosexual men theoretically could have been followed for 7 years based on their estimated date of seroconversion and the ultimate date of censoring, regardless of their clinical outcome. The clinical, vital and follow-up status for these IDU and homosexual men at 7 years after seroconversion were as follows: Twenty-seven IDU (12.1\%) and 116 homosexual men (34.2\%) developed AIDS, and 24 IDU (10.7\%) and five homosexual men (1.5\%) died without an AIDS diagno- 


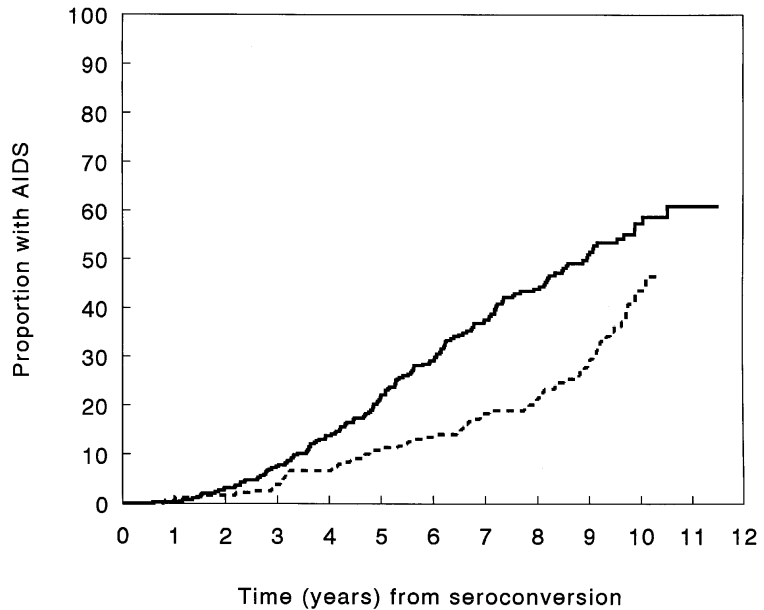

Fig. 1. Kaplan-Meier estimates of the cumulative proportion deceased among injecting drug users (--) and homosexual men (-) registered in the European Seroconverter Study and the Tricontinental Seroconverter Study, respectively. Deaths from all causes were taken into account. Curves are truncated when fewer than five subjects remain at risk.

sis. A total of 55 AIDS-free IDU (24.6\%) and 55 homosexual men $(16.2 \%)$ were considered lost to follow-up because their immunological status could not be determined due to missing or limited CD4 data. The median number of CD4 counts used in the linear least square regression analysis was nine (IQR five-12) for 118 IDU (52.7\%) and 12 (IQR nine-22) for 163 homosexual men (48.1\%) with three or more CD4 counts. The numbers and proportions of subjects classified as non-progressors are displayed in Table 4. As in a previously published analysis on non-progression in the Tricontinental Seroconverter Study [16], we excluded subjects who were lost to follow-up or died without AIDS from the denominator of the proportions. However, because losses to follow-up and pre-AIDS deaths are more common among IDU than homosexual men, proportions are also presented with inclusion of all subjects who theoretically could have had 7 years of follow-up in the denominator. The definition applied identified IDU as having the highest proportion of non-progressors. This definition classified 15.2 and $9.8 \%$ of IDU and 7.5 and $6.2 \%$ of homosexual men as non-progressors, 7 years following seroconversion when losses to follow-up and pre-AIDS deaths were excluded and included in the denominator of the proportion, respectively. Risk group differences decreased and became insignificant when losses to follow-up and pre-AIDS mortality were included in the denominator.

In addition, we evaluated two definitions that required the subject's last available CD4 determination to be $>$ 500 cells $\times 10^{6} / 1[13]$ and the 7-year calculated CD4 count based on a linear least-square regression line to be $>500$ cells $\times 10^{6} / 1$ [16], respectively. These definitions yielded higher proportions of non-progressors

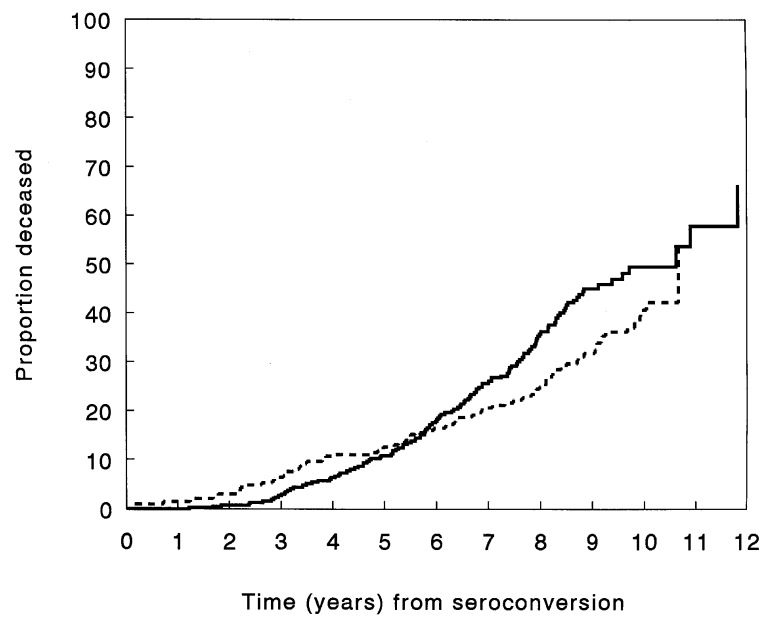

Fig. 2. Kaplan-Meier estimates of the cumulative proportion with AIDS among injecting drug users (--) and homosexual men (-) registered in the European Seroconverter Study and the Tricontinental Seroconverter Study, respectively. Curves are truncated when fewer than five subjects remain at risk.

Table 4. Numbers and proportions of non-progressors* among injecting drug users (IDU) and homosexual men in the European Seroconverter Study and the Tricontinental Seroconverter Study, respectively.

\begin{tabular}{lll}
\hline & $\mathrm{n} /$ total & $\%$ \\
\hline $\begin{array}{l}\text { Losses to follow-up and pre-AIDS } \\
\text { deaths excluded from the denominator }\end{array}$ & & \\
Risk group & & \\
$\quad$ IDU & $22 / 145$ & $15.2 \%$ \\
$\quad$ Homosexual men & $21 / 279$ & $7.5 \%$ \\
$P$ value $^{+}$ & 0.02 & \\
$\quad$ Losses to follow-up and pre-AIDS & & \\
deaths included in the denominator & & \\
Risk group & & \\
IDU & & \\
$\quad$ Homosexual men & $22 / 224$ & $9.8 \%$ \\
$P$ value* $^{*}$ & $21 / 339$ & $6.2 \%$ \\
\hline
\end{tabular}

*AIDS-free and a positive slope at 7 years after seroconversion.

${ }^{+} P$ value by $\chi^{2}$ test comparing IDU with homosexual men.

among homosexual men than among IDU (data not shown), but did not change considerably the results on risk group differences. For both IDU and homosexual men, about $5 \%$ of the subjects were identified as nonprogressors by the criteria of all three definitions and risk group differences were not significant, regardless of the denominator.

\section{Discussion}

Combining data on seroconverters followed in 12 cohorts of IDU or homosexual men enabled us to evaluate risk group variation in progression and non-progression in a large group without bias due to unknown duration of infection. The major finding of the present 
study is that our data do not show notable effects of risk group on the progression to AIDS and death from AIDS, and non-progression. In contrast, pre-AIDS mortality was much higher in IDU than homosexual men, partly due to a high proportion of suicide and overdose deaths among IDU.

Our results concerning the effect of risk group on progression to AIDS are in agreement with the results of other studies among IDU and homosexual men with documented dates of seroconversion, showing that IDU do not progress at a substantially slower or faster rate $[2-6,8]$. Only one study combining data from five cohorts found that homosexual men had a twofold higher risk of developing AIDS than IDU after adjusting for potential confounders [9], and one cannot exclude the possibility that heterogeneity in the methods used to estimate seroconversion dates across the participating cohorts might have biased their estimate associated with risk group.

We examined whether the small difference in progression to AIDS between IDU and homosexual men could be explained by the occurrence of Kaposi's sarcoma, which is known to be an early AIDS diagnosis, and is much more common among homosexual men than IDU. Ignoring Kaposi's sarcoma, progression rates were similar. Because we did not have complete information on AIDS conditions following the primary AIDS-defining event, subjects with Kaposi's sarcoma were censored in the same manner as censoring due to withdrawal. In doing so, the assumption of independency of censoring and the outcome AIDS might be violated and the hazard for progression to AIDS might be underestimated for homosexual men. However, other studies among seroconverters $[2,4,22,36]$, of which some did have information on the occurrence of second AIDS-defining illnesses, also showed that when Kaposi's sarcoma is ignored as an AIDS-defining illness, progression rates were similar between homosexual men and other risk groups.

In the present analysis, AIDS was defined by the 1987 CDC criteria [26]. In 1993, recurrent pneumonia and pulmonary tuberculosis were added to the AIDS case definition [37]. Because these infectious diseases are more common in IDU than in homosexual men [38-40], progression rates to AIDS are expected to appear even more similar in the near future.

Only one other published study has compared the progression from seroconversion to death between cohorts of IDU and homosexual men [7]. Contrary to our findings, the Kaplan-Meier curves of progression to death from any cause did not cross in this study. An explanation for the conflicting results of both studies might be that pre-AIDS deaths and deaths from AIDS were more frequent among homosexual men and less frequent among IDU than in our study.

Most studies among seroconverters showed significantly faster progression rates to AIDS or death with increasing age [6,41-45], but others could not confirm this trend $[2,17]$, probably due to a narrow age distribution. It is noteworthy that our data did not indicate a linear effect of age. We found that subjects younger than 24 years progressed more slowly than older subjects. For the older subgroups we did not find a significant effect of age. A comparable trend has been demonstrated by two studies $[4,46]$, in which no differences in progression between subgroups of young and middle-aged subjects could be demonstrated, but relatively old subjects (above about 44 and 50 years, respectively), which are not common in our study, were found to be at increased risk.

Gender differences in progression did not confound our observed small effect of risk group. Consistent with our observations, several previous studies reported a very marginal effect of gender among IDU $[17,41,43]$.

In the analysis of progression to AIDS and death from AIDS, we regarded pre-AIDS death as a censoring event. However, non-AIDS mortality is higher for HIV-positive IDU than for HIV-negative IDU [47-50] and pre-AIDS mortality rates among HIV-infected IDU increase with decreasing CD 4 count [51]. Therefore, independence of pre-AIDS death and (death from) AIDS is unlikely and the estimates of risk group are expected to be biased from unity, as has been shown for the two Amsterdam cohorts [2].

Despite left truncation, the inclusion of retrospectively identified seroconverters causes overestimates of progression rates if fast progressors are over-represented, which seems likely for clinically based cohort studies. On the other hand, progression rates are underestimated if slow progressors are over-represented among retrospectively identified seroconverters, which seems likely for cohort studies that required subjects to be symptom-free at study entry. Therefore and because retrospectively identified seroconverters were more frequent among IDU than among homosexual men, we repeated our analysis for those who seroconverted after study entry or, at the most, 1 year before study entry. The small increase in the RH of progression for homosexual men compared with IDU indicate that fast progressors, on average, are slightly over-represented among IDU for whom seroconversion was determined retrospectively.

We applied two censoring strategies and found that the hazard associated with risk group was higher for the strategy that censored event-free subjects that had not been seen within a year of the ultimate censoring date at the last follow-up visit, in comparison with the strategy that censored event-free subjects not known to be 
lost to follow-up at the ultimate date. It appeared that homosexual men progressed more rapidly using the former censoring strategy compared to the latter strategy. Probably not all AIDS cases and deaths are captured by follow-up procedures, and censoring all subjects not known to have developed AIDS, to be deceased or to be lost to follow-up at the ultimate censoring date might be too optimistic. For IDU, differences between both censoring strategies were small. Due to the fact that some IDU cohorts were not able to match with AIDS and deaths registers, censoring was already more conservative for IDU. Moreover, IDU had a shorter follow-up period than homosexual men, and therefore missed events will have less effect. Another explanation may be that studies among IDU put more efforts in extensive follow-up procedures because IDU are known to drop out frequently.

Risk group differences in non-progression were studied by evaluating clinical and immunological criteria of non-progression. The difficulty of using definitions based on absolute CD4 counts is that CD4 lymphocyte levels are known to vary widely within and between individuals and laboratories, and may fluctuate depending on smoking, use of antiretroviral therapy, diurnal variation and being splenectomized. Therefore, a definition based on a positive slope is expected to be more consistent across cohorts and risk groups provided that the number and timing of CD4 determinations are more or less equal. Applying this definition, we found higher proportions of non-progressors among IDU than among homosexual men. The higher proportions among IDU for this definition might be explained by differential timing of CD4 determinations. Relative to IDU, homosexual men had more CD4 determinations in the first few years following seroconversion (data not shown) and subsequently homosexual men would be more likely to have a negative slope.

Withdrawal differed between the two risk groups. Therefore, inclusion or exclusion of losses to follow-up and pre-AIDS deaths in the denominator altered results. By excluding losses to follow-up and pre-AIDS mortality from the denominator, we assume that withdrawal is independent of non-progression. As previously mentioned, pre-AIDS mortality may not be independent of progression or non-progression. Furthermore, it will depend on whether those who were not actively followed had a faster immunological progression than those who remained in the study. We expect the 'true' proportion to be in between both estimates. However, when taking the most stringent criteria for non-progression (i.e., based on both slope and absolute CD4 cell count), the proportions of nonprogressors were very similar for IDU and homosexual men, regardless of the denominator.

Limitations on our study include the multicohort design with differences in recruitment, selection criteria, setting of follow-up, frequency of follow-up visits, follow-up procedures, etc. Furthermore, the effect of risk group could not be controlled for site differences because only Amsterdam provided data on both risk groups. Finally, we were not able to adjust for other potential confounders (e.g., symptomatic primary HIV infection, coinfections, HIV treatment, drug use and sexual behaviour).

We conclude that little evidence was found for differences in the rate of HIV disease progression or nonprogression between IDU and homosexual men. This suggests that the mode of acquisition does not play a major role in pathogenesis. However, pre-AIDS mortality and a non-linear effect of age should be considered in planning interventions, as well as in studies comparing risk groups and modelling the epidemic. Further studies comparing risk groups should also consider differences in the spectrum of AIDS-defining conditions, and the influence of censoring strategies, the length of the seroconversion interval, inclusion of retrospectively versus prospectively identified seroconverters and loss to follow-up.

\section{Acknowledgements}

The authors thank J.S. Bax and L. Gerber for their helpful assistance, and R.B. Geskus for estimating the seroconversion dates.

\section{References}

1. Brookmeyer R, Gail MH, Polk BF: The prevalent cohort study and the acquired immunodeficiency syndrome. Am J Epidemiol 1987, 126:14-24.

2. Spijkerman IJB, Langendam MW, Veugelers PJ, et al.: Differences in progression to AIDS between injection drug users and homosexual men with documented dates of seroconversion. Epidemiology 1996, 7:571-577.

3. Pezzotti $P$, Rezza G, Lazzarin $A$, et al.: Influence of gender, age and transmission category on the progression from HIV seroconversion to AIDS. J Acquir Immune Defic Syndr 1992, 5:745-747.

4. Biggar RJ, the International Registry of Seroconverters: AIDS incubation in 1891 HIV seroconverters from different exposure groups. AIDS 1990, 4:1059-1066.

5. Mariotto AB, Mariotti S, Pezzotti P, Rezza G, Verdecchia A: Estimation of the acquired immunodeficiency syndrome incubation period in intravenous drug users: a comparison with male homosexuals. Am J Epidemiol 1992, 135:428-437.

6. Gauvreau K, DeGruttola V, Pagano M, Bellocco R: The effect of covariates on the induction time of AIDS using improved imputation of exact seroconversion times. Stat Med 1994, 13:2021-2030.

7. Multicohort Analysis Project Workshop: Extending public health surveillance of HIV infection: information from a five cohort workshop. Stat Med 1993, 12:2065-2085.

8. Galai N, Vlahov D, Margolick JB, Chen K, Graham NMH, Muñoz A: Changes in markers of disease progression in HIV-1 seroconverters: a comparison between cohorts of injecting drug 
users and homosexual men. J Acquir Immune Defic Syndr Hum Retrovirol 1995, 8:66-74.

9. Multicohort Analysis Project Workshop: Immunologic markers of AIDS progression: consistency across five HIV-infected cohorts. Part I. AIDS 1994, 8:911-921.

10. Keet IPM, Krol A, Klein MR, et al.: Characteristics of long-term asymptomatic infection with human immunodeficiency virus type 1 in men with normal and low CD4+ cell counts. J Infect Dis 1994, 169:1236-1243.

11. Sheppard HW, Lang W, Ascher MS, Vittinghoff E, Winkelstein W: The characterization of non-progressors: long-term HIV-1 infection with stable CD4+ T-cell levels. AIDS 1993 7:1159-1166.

12. Muñoz A, Kirby AJ, He YD, et al.: Long-term survivors with HIV-1 infection: incubation period and longitudinal patterns of CD4+ Iymphocytes. J Acquir Immune Defic Syndr 1995, 8:496-505.

13. Buchbinder SP, Katz MH, Hessol NA, O'Malley PM, Holmberg SD: Long-term HIV-1 infection without immunologic progression. AIDS 1994, 8:1123-1128.

14. Strathdee SA, Craib KJP, Hogg RS, O'Shaughnessy MVO, Montaner JSG, Schechter MT: Long-term non-progression in HIV infection [letter]. Lancet 1995, 346:1372.

15. Phillips AN, Sabin CA, Elford J, Bofill M, Janossy G, Lee CA: Use of CD4 lymphocyte count to predict long term survival free of AIDS after HIV infection. Br Med J 1994, 309:309-313.

16. Strathdee SA, Veugelers PJ, Page-Shafer KA, et al.: Lack of consistency between five definitions of nonprogression in cohorts of HIV-infected seroconverters. AIDS 1996, 10:959-965.

17. Brettle RP, McNeil AJ, Burns S, et al.: Progression of HIV: follow-up of Edinburgh injecting drug users with narrow seroconversion intervals in 1983-1985. AIDS 1996, 10:419-430.

18. Skidmore CA, Robertson JR, Robertson AA, Elton RA: After the epidemic: follow up study of HIV seroprevalence and changing patterns of drug use. Br Med J 1990, 300:219-223.

19. Brettle RP, Bisset K, Burns S, et al.: Human immunodeficiency virus and drug misuse: the Edinburgh experience. $\mathrm{Br}$ Med J 1987, 295:421-424.

20. van den Hoek JAR, Coutinho RA, van Haastrecht HJA, van Zadelhoff AW, Goudsmit J: Prevalence and risk factors of HIV infections among drug users and drug-using prostitutes in Amsterdam. AIDS 1988, 2:55-60.

21. Ledergerber B, Von Overbeck J, Egger $M$, Luthy R: The Swiss HIV Cohort Study: rationale, organization and selected baseline characteristics. Soz Praventivmed 1994, 39:387-394.

22. Carré N, Deveau C, Belanger F, et al.: Effect of age and exposure group on the onset of AIDS in heterosexual and homosexual HIV-infected patients. AIDS 1994, 8:797-802.

23. Goldberg DJ, Emslie JA, Smyth W, Reid D, collaborating microbiologists: A system for surveillance of voluntary HIV testing: results of the first 2 years 1989-1990. AIDS 1992, 6:495-500.

24. Zangerle $R$, Fuchs D, Rossler $H$, et al.: Trends in HIV infection among intravenous drug users in Innsbruck, Austria. J Acquir Immune Defic Syndr 1992, 5:865-871.

25. Veugelers PJ, Page KA, Tindall B, et al.: Determinants of HIV disease progression among homosexual men registered in the Tricontinental Seroconverter Study. Am J Epidemiol 1994 140:747-758

26. Centers for Disease Control: Revision of the CDC surveillance case definition for acquired immunodeficiency syndrome. MMWR 1987, 6:1S-15S.

27. Veugelers PJ, Strathdee SA, Moss AR, et al: Is the human immunodeficiency virus-related Kaposi's sarcoma epidemic coming to an end? Insights from the Tricontinental Seroconverter Study. Epidemiology 1995, 6:382-386.

28. Law CG, Brookmeyer R: Effects of mid-point imputation on the analysis of doubly censored data. Stat Med 1992 11:1569-1578.

29. Jongbloed G: The Iterative Convex Minorant Algorithm for Nonparametric Estimation. Technical Report. Delft: Delft University of Technology, 1995.

30. Groeneboom P, Wellner JA: Information Bounds and Non-parametric Maximum Likelihood Estimation. Berlin: Birkhäuser Verlag, 1992.

31. van Haastrecht HJA, van den Hoek JAR, Coutinho RA: High mortality among HIV-infected injecting drug users without AIDS-diagnosis: implications for HIV infection epidemic modellers? AIDS 1994, 8:363-366.

32. Lang W, Perkins $H$, Anderson R, et al.: Patterns of T-lymphocyte changes with human immunodeficiency virus infection: from seroconversion to the development of AIDS. J Acquir Immune Defic Syndr 1989, 2:63-69.

33. Margolick JB, Donnenberg AD, Muñoz A, et al:: Changes in $\mathbf{T}$ and non-T lymphocyte subsets following seroconversion to HIV-1: stable CD3+ and declining CD3-populations suggest regulatory responses linked to loss of CD4 lymphocytes. J Acquir Immune Defic Syndr 1993, 6:153-161.

34. Beral V, Peterman TA, Berkelman RL, Jaffe HW: Kaposi's sarcoma among persons with AIDS: a sexually transmitted infection? Lancet 1990, 335:123-128.

35. Crowe SM, Carlin JB, Stewart KI, Lucas R, Hoy JF: Predictive value of CD4-lymphocyte numbers for the development of opportunistic infections and malignancies in HIV-infected persons. J Acquir Immune Defic Syndr 1991, 4:770-776.

36. Operskalski EA, Stram DO, Lee H, Transfusion Safety Study Group: Human immunodeficiency virus type 1 infection: relationship of risk group and age to rate of progression to AIDS. J Infect Dis 1995, 172:648-655.

37. Centers for Disease Control and Prevention: Revised classification system for HIV infection and expanded surveillance case definition for AIDS among adolescents and adults. MMWR 1992, 41 (RR-17):1-19.

38. Mientjes GHC, van Ameijden EJC, Keet IPM, van Deutekom H, van den Hoek JAR, Coutinho RA: Disproportional impact of the revised AIDS surveillance definition on the AIDS incidence among drug users compared to homosexual men. Eur J Publ Health 1995, 5:288-290.

39. Dupon $M$, Texier-Maugein J, Leroy $V$, et al.: Tuberculosis and HIV infection: a cohort study of incidence and susceptibility to antituberculous drugs, Bordeaux, 1985-1993. AIDS 1995, 9:577-583.

40. Hirschtick RE, Glassroth J, Jordan MC, et al.: Bacterial pneumonia in persons infected with the human immunodeficiency virus. N Engl J Med 1995, 333:845-851.

41. Ronald PJM, Robertson JR, Elton RA: Continued drug use and other cofactors for progression to AIDS among injecting drug users. AIDS 1994, 8:339-343.

42. Rosenberg PS, Goedert JJ, Biggar RJ, Multicenter Hemophilia Cohort Study and the International Registry of Seroconverters: Effect of age at seroconversion on the natural AIDS incubation distribution. AIDS 1994, 8:803-810.

43. The Italian Seroconversion Study: Disease progression and early predictors of AIDS in HIV-seroconverted injecting drug users. AIDS 1992, 6:421-426.

44. Hessol NA, Koblin BA, van Griensven GJP, et al.: Progression of human immunodeficiency virus type 1 (HIV-1) infection among homosexual men in hepatitis $B$ vaccine trial cohorts in Amsterdam, New York City, and San Francisco, 1978-1991. Am J Epidemiol 1994, 139:1077-1087.

45. Darby SC, Ewart DW, Giangrande PLF, Spooner RJD, Rizza CR, the UK Heamophilia Centre Directors' Organisation: Importance of age at infection with HIV-1 for survival and development of AIDS in UK haemophilia population. Lancet 1996, 347:1573-1579.

46. Martin JN, Colford JM, Ngo L, Tager IB: Effect of older age on survival in human immunodeficiency virus (HIV) disease. $\mathrm{Am} \mathrm{J}$ Epidemiol 1995, 142:1221-1230.

47. Selwyn PA, Alcabes P, Hartel D, et al.: Clinical manifestations and predictors of disease progression in drug users with human immunodeficiency virus infection. N Engl J Med 1992, 327:1697-1703.

48. Goedert JJ, Pizza G, Gritti FM, et al:: Mortality among drug users in the AIDS era. Int J Epidemiol 1995, 24:1204-1210.

49. Zaccarelli M, Gattari P, Rezza G, et al.: Impact of HIV infection on non-AIDS mortality among Italian injecting drug users. AIDS 1994, 8:345-350.

50. Stoneburner RL, Des Jarlais DC, Benezra D, et al.: A larger spectrum of severe HIV-1 related disease in intravenous drug users in New York City. Science 1988, 242:916-919.

51. van Haastrecht HJA, van Ameijden EJC, van den Hoek JAR, Mientjes GHC, Bax JS, Coutinho RA: Predictors of mortality in the Amsterdam cohort of HIV-positive and HIV-negative drug users. Am J Epidemiol 1996, 143:380-391. 


\section{Appendix}

The following investigators participate in the European Seroconverter Study in injecting drug users: J.R. Robertson for the Edinburgh Drug Addiction Study; R.P. Brettle and A.M. Richardson for the Edinburgh City Hospital Cohort Study; J.A.R. van den Hoek (project leader) and R.A. Coutinho for the Amsterdam Cohort Study among drug users; B. Hirschel, B. Broers, and H. McArdle for the Geneva HIV Cohort Study; D.J. Goldberg and J. McMenamin for the Scottish National Collaborative HIV Testing Study; N. Carré for the French SEROCO Study; R. Zangerle for the Innsbruck AIDS Study, I. Hernández Aguado for the Valencian HIV Seroconversion Study; D.A. Hawkins for the London St. Stephan's Clinic IV study.

The following investigators participate in the Tricontinental Seroconverter Study in homosexual men: M.T. Schechter, S.A. Strathdee and K.J.P. Craib for the Vancouver Lymphadenopathy-AIDS Study; J.M. Kaldor, A. McNulty, J. Vizzard and D.A. Cooper for the Sydney AIDS Prospective Study; G.J.P. van Griensven (project leader) and R.A. Coutinho for the Amsterdam Cohort Study among homosexual men; A.R. Moss and E. Charlebois for the San Francisco General Hospital Cohort; D. Osmond, W. Winkelstein $\mathrm{Jr}$ and K.A. Page-Shafer for the San Francisco Men's Health Study. 